\title{
Characterization of the developing small intestine in the absence of either GATA4 or GATA6
}

\author{
Emily M Walker, Cayla A Thompson, Bridget M Kohlnhofer, Mary L Faber and Michele A Battle*
}

\begin{abstract}
Background: Studies of adult mice lacking either GATA4 or GATA6 in the small intestine demonstrate roles for these factors in small intestinal biology. Deletion of Gata4 in the adult mouse intestine revealed an essential role for GATA4 in jejunal function. Deletion of Gata6 in the adult mouse ileum alters epithelial cell types and ileal enterocyte gene expression. The effect of deletion of Gata4 or Gata6 alone during embryonic small intestinal development, however, has not been examined. We recently demonstrated that loss of both factors in double conditional knockout embryos causes severe defects in jejunal development. Therefore, the goal of this study is to provide phenotypic analysis of the small intestine of single Gata4 and Gata6 conditional knockout embryos.

Results: Villin-Cre was used to delete Gata4 or Gata6 in the developing intestinal epithelium. Elimination of either GATA4 or GATA6 in the jejunum, where these factors are co-expressed, caused changes in enterocyte and enteroendocrine cell gene expression. Ectopic expression of markers of the ileal-specific bile acid metabolism pathway was induced in GATA4-deficient jejunum but not in GATA6-deficient jejunum. A subtle increase in goblet cells was also identified in jejunum of both mutants. In GATA6-deficient embryonic ileum, villus length was altered, and enterocyte gene expression was perturbed including ectopic expression of the colon marker Car1. Goblet cells were increased, and enteroendocrine cells were decreased.
\end{abstract}

Conclusions: Overall, we show that aspects of the phenotypes observed in the small intestine of adult Gata4 and Gata6 conditional knockout mice emerge during development. The effect of eliminating GATA6 from the developing ileum was greater than that of eliminating either GATA4 or GATA6 from the developing jejunum likely reflecting functional redundancy between these factors in the jejunum. Although GATA4 and GATA6 functions overlap, our data also suggest unique functions for GATA4 and GATA6 within the developing intestine. GATA4 likely operates independently of GATA6 within the jejunum to regulate jejunal versus ileal enterocyte identity and consequently jejunal physiology. GATA6 likely regulates enteroendocrine cell differentiation cell autonomously whereas GATA4 affects this population indirectly.

Keywords: GATA4, GATA6, Small intestine, Development, Epithelium, Conditional knockout

\section{Background}

The zinc-finger DNA binding transcription factors GATA4 and GATA6 are expressed in the small intestinal epithelium throughout development and adulthood [1-7]. GATA4, unlike GATA6, is present in a restricted pattern in the adult small intestine; it is expressed in the proximal small intestine (duodenum and jejunum) but absent from the distal small intestine (ileum) $[1,4,8]$. Global Gata4 or Gata6 knockout causes early embryonic lethality necessitating conditional knockout (cKO) strategies to analyze

\footnotetext{
* Correspondence: mbattle@mcw.edu

Department of Cell Biology, Neurobiology and Anatomy, Medical College of Wisconsin, 8701 Watertown Plank Road, Milwaukee, WI 53226, USA
}

their roles in organogenesis [5,9-11]. Several studies performed by our laboratory and others to eliminate GATA4 or GATA6 specifically in the intestinal epithelium have uncovered roles for these factors in small intestinal biology $[1,2,4]$. Fat and cholesterol absorption are disrupted in adult mice lacking GATA4 in the jejunal epithelium [1]. Moreover, expression of many jejunal-specific transcripts is lost and expression of many ileal-specific transcripts is induced in GATA4-deficient jejunum demonstrating a role for GATA4 in regulating jejunal versus ileal intestinal identity [1,4]. Although constitutive Villin-Cre has been used to delete Gata4 in the small intestine during 
development [1], GATA4-deficient embryonic intestine was not examined.

Unlike Gata4 cKO adult mice, elimination of Gata6 from the adult jejunal epithelium using tamoxifen-inducible Villin-Cre does not decrease expression of jejunal enterocyte markers or induce expression of ileal enterocyte markers suggesting that jejunal identity is maintained in its absence [2]. Increased Paneth cells with atypical granules are reported in GATA6-deficient jejunum [2]. In contrast, loss of Gata6 from the adult ileum, a tissue lacking GATA4, results in shortened villi, reduced proliferative, enteroendocrine, and Paneth cells, and increased crypt goblet cells [2]. Changes in ileal enterocyte gene expression also occur; small intestinal enterocyte marker expression is decreased, and colonocyte marker expression is induced suggesting that GATA6 plays a role in regulating intestinal identity in the distal small intestine [2]. These studies did not induce Gata6 deletion during embryonic development precluding analysis of embryonic intestine.

We recently demonstrated that simultaneous deletion of both Gata4 and Gata6 within the developing intestinal epithelium using constitutive Villin-Cre severely disrupts jejunal development causing Gata4-Gata6 double cKO mice to die within a day of birth [12]. Intestinal epithelial architecture is altered in the absence of both GATA4 and GATA6 with the jejunum of Gata4-Gata6 double cKO embryos containing short, blunted villi. Furthermore, differentiated epithelial cell populations are skewed in Gata4-Gata6 double cKOs. Enterocytes are decreased and goblet and proliferative cells are increased in mutant jejunum. The effect of deletion of Gata4 or Gata6 alone during embryonic development of the small intestine, however, has not been examined. Therefore, the goal of this study is to provide phenotypic analysis of intestinal development in single Gata4 and Gata6 cKO embryos derived using constitutive Villin-Cre. We examined the jejunum of Gata4 Villin-Cre and Gata6 Villin-Cre cKO embryos and the ileum of Gata6 Villin-Cre cKO embryos at E18.5. We found that jejunum lacking either GATA4 or GATA6 was largely normal. Changes in enterocyte gene expression reflecting a transition from jejunal to ileal identity were identified only in GATA4 mutant jejunum. Analysis of GATA6 mutant ileum revealed a phenotype similar to that observed when GATA6 is eliminated from the adult ileal epithelium. We observed shortened villi and subtle changes in epithelial cell populations including altered enterocyte gene expression, decreased enteroendocrine cells, and increased goblet and proliferative cells.

\section{Results and discussion}

To determine GATA4 or GATA6 function during small intestinal development, we generated intestinal epitheliumspecific Gata4 and Gata6 cKO embryos using the following mouse lines: Gata4 ${ }^{\text {tm1.1Sad }}\left(\right.$ Gata $^{\text {loxP }}$ ), Gata $4^{\text {tmolEno }}$ $\left(\right.$ Gata4 $\left.^{-}\right)$, Gata6 $^{\text {tm2.1Sad }}\left(\right.$ Gata6 $\left.^{\text {loxP }}\right)$, Gata6 ${ }^{\text {tm2.2Sad }}\left(\right.$ Gata6 $\left.^{-}\right)$, and $\mathrm{Tg}$ (Vil-cre)997Gum (Villin-Cre) [10,13-15]. For our studies, control embryo genotypes were Gata4 $4^{\text {loxP/+ }}$ or Gata6 $^{\text {loxP/+ }}$ and experimental embryo genotypes were Gata4 $^{\text {loxP/- Villin-Cre or Gata6 }}{ }^{\text {loxP/- Villin-Cre. Although }}$ Villin-Cre is widely used to generate genetic deletions within the intestinal epithelium, the time course of Villin-Cre activity within the embryonic small intestinal epithelium has not been shown. Therefore, we mated Villin-Cre mice with the Rosa26 conditional LacZ reporter strain Gt(ROSA)26Sor ${ }^{\text {tm1Sor }}$ (Rosa26R) [16] and stained embryonic intestine for $\beta$-galactosidase activity between E13.5-E15.5. We found minimal Cre activity present at E13.5 (Figure 1). By E14.5, the intestinal epithelium of most embryos analyzed (7/11) showed robust Cre activity (Figure 1). A small subset of E14.5 embryos (4/11)

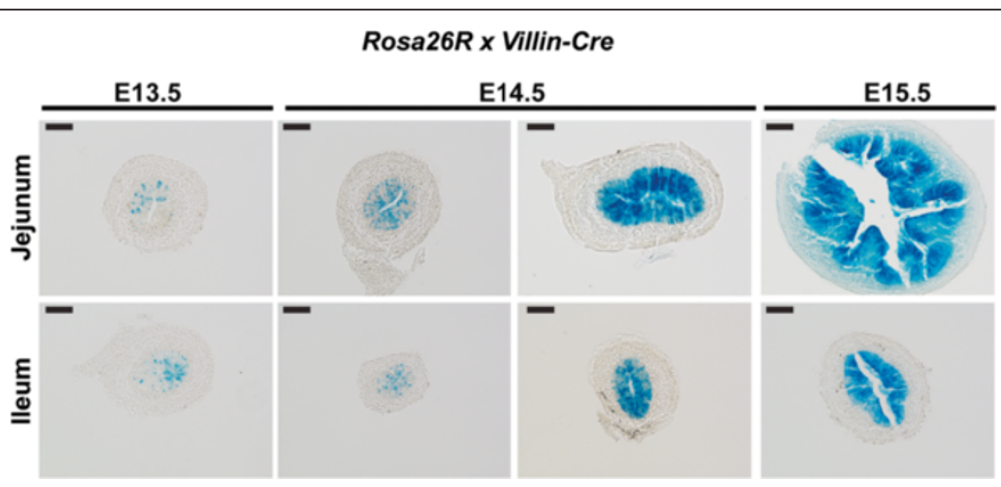

Figure 1 Villin-Cre shows maximal activity between E14.5-E15.5. Villin-Cre (Tg(Vil-cre)997Gum) and Rosa26R mice (Gt(ROSA)26Sor $\left.{ }^{\text {tm } 150 r}\right)$ were crossed to determine Cre activity. Embryonic small intestine harvested at E13.5, E14.5, and E15.5 was stained for $\beta$-galactosidase activity (blue staining) to identify Cre activity. At E13.5, minimal Cre activity was detected in jejunum and ileum ( $n=9$ Cre +embryos from 2 litters; $5 / 9$ showed scattered blue cells; $4 / 9$ had no blue cells). At E14.5, two types of staining were detected ( $n=11$ Cre + embryos from 2 litters). One group (4/11) had heterogeneous staining in jejunum and ileum (E14.5 left panel). The other group (7/11) had robust homogeneous staining in jejunum and ileum (E14.5 right panel). At E15.5, we observed 3/4 Cre + embryos with robust homogeneous staining in jejunum and ileum. One Cre + embryo had a low level of blue staining reminiscent of earlier stages ( $n=4$ Cre + embryos from 1 litter). Scale bars, $50 \mu \mathrm{m}$. 
had heterogeneous intestinal Cre activity (Figure 1). By E15.5, the majority of embryos examined (3/4) showed robust intestinal Cre activity (Figure 1). Therefore, we conclude that the Villin promoter drives robust Cre recombinase activity in the small intestinal epithelium by E14.5-15.5 (Figure 1). The heterogeneity in Cre activity we observed at E14.5 and E15.5 could reflect differences in Cre activity itself or in the developmental maturity of embryos within litters.

Although GATA4 is absent from the distal small intestine of late stage embryos (E17.5) and adult mice [1,3,4,8], the time at which restricted intestinal GATA4 expression is first detectable is unknown. The relationship between GATA4 restriction and onset of Villin-Cre activity is relevant to our study to identify which regions of the gut will be affected by Gata4 deletion. Therefore, to determine the earliest time at which GATA4 expression is restricted, we isolated the distal most $0.5 \mathrm{~cm}$ of mouse embryonic small intestinal tissue at various gestational ages and used immunohistochemistry to identify GATA4. At E12.5, GATA4 positive cells were found throughout the distal small intestine (Figure 2A, B). Although most cells within this region expressed GATA4, we did identify some GATA4 negative cells (Figure 2B). By E13.5, we observed

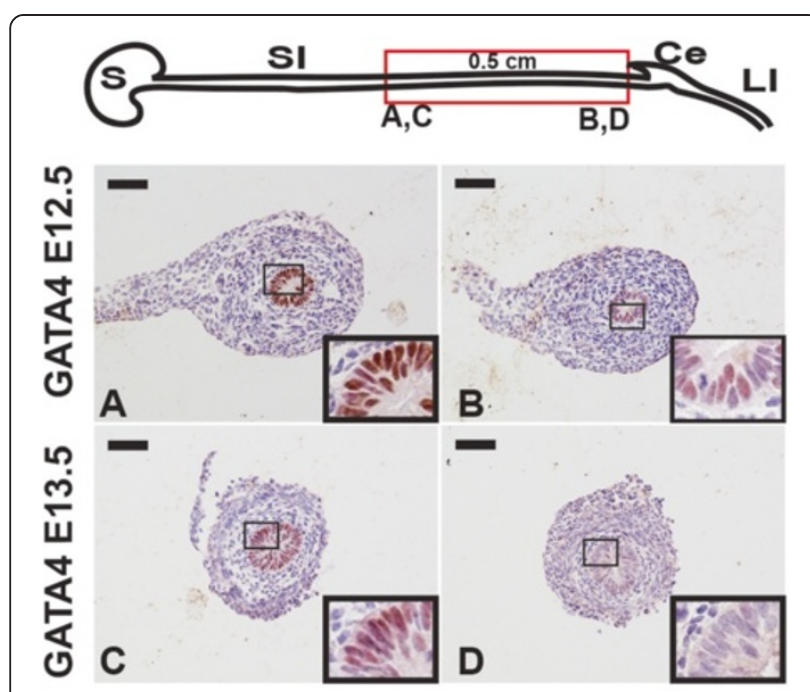

Figure 2 GATA4 is excluded from the distal small intestinal epithelium during early development. Immunohistochemistry (IHC) for GATA4 (brown nuclear stain) is shown in tissue from the distal region of small intestine of E12.5 $(n=3)$ and E13.5 $(n=7)$ CD1 embryos. A $0.5 \mathrm{~cm}$ piece of small intestine dissected just superior to the cecum (red box) from each embryo was sectioned through its entirety and stained for GATA4. Tissue was also counterstained with hematoxylin. GATA4 was present throughout the region examined at E12.5 (panels $\mathbf{A}$ and $\mathbf{B}$ ) although scattered cells within the most distal section of the tissue (panel $\mathbf{B}$ ) had already lost GATA4 expression. By E13.5, GATA4 protein was absent from all cells in the most distal region of the small intestine (compare panels $\mathbf{C}$ and $\mathbf{D}$ ). Insets show higher magnification images of boxed regions. S, stomach; Sl, small intestine; Ce, cecum; LI, large intestine. Scale bars, 50 m. loss of GATA4 from all cells of the most distal region of the small intestine (Figure 2C, D). Therefore, we conclude that GATA4 is initially expressed throughout the small intestinal epithelium and that it becomes excluded from the distal small intestinal epithelium at E12.5-E13.5. Moreover, because Villin-Cre did not become maximally activated until E14.5-E15.5, we conclude that Gata4 knockout using Villin-Cre should not impact development of the distal small intestine (ileum) as GATA4 is lost in this region prior to Cre activity. Therefore, we limited our analysis of Gata4 cKO embryos to jejunum. Because GATA6 is expressed throughout the small intestinal epithelium $[2,8]$, we analyzed both jejunum and ileum of Gata6 cKO embryos. We validated loss of GATA4 or GATA6 protein in small intestine from E18.5 Gata4 cKO and Gata6 cKO embryos using immunohistochemistry (Figure 3A). Moreover, we examined tissue lacking GATA4 or GATA6 to determine if expression of the remaining GATA factor changed. We found that GATA4 was maintained in GATA6-deficient jejunum and that GATA6 was maintained in GATA4-deficient jejunum (Figure 3B). Finally, GATA4 was not induced in GATA6-deficient ileum (Figure 3B). Analysis of Gata4 and Gata6 mRNA levels by qRT-PCR confirmed these results (Additional file 1).

To identify the impact of loss of either GATA4 or GATA6 on jejunal epithelial development, we examined jejunum of Gata4 and Gata6 cKO embryos at E18.5. We chose to analyze GATA-deficient intestine at E18.5 because developmental processes such as villus morphogenesis and epithelial cytodifferentiation are essentially complete by this time [17], and our goal is to determine the extent to which either GATA4 or GATA6 are required to complete these processes. We recognize, however, that because the onset of Villin-Cre activity occurs earlier than E18.5, the effects that we observe in GATA mutants may represent both primary and secondary consequences of Gata4 or Gata6 deletion. To examine epithelial architecture in jejunum lacking GATA4 or GATA6, we used hematoxylin and eosin (H\&E) staining and found GATA mutants to be indistinguishable from controls (Figure 4A). Because we have previously demonstrated that jejunal villus length is significantly decreased in adult Gata4 cKO animals [1], we determined average villus length in control and mutant jejunal tissue. We found no difference in jejunal villus length among Gata4 cKO, Gata6 cKO, and control embryos (Figure 4B). Therefore, we conclude that GATA4 exerts its effect on jejunal villus length after birth. In the mouse, small intestinal development continues during the postnatal period with substantial remodeling of the mucosa occurring including replacement of intervillus regions by crypts [17]. It is possible that GATA4 acts during these processes to effect final villus length. 


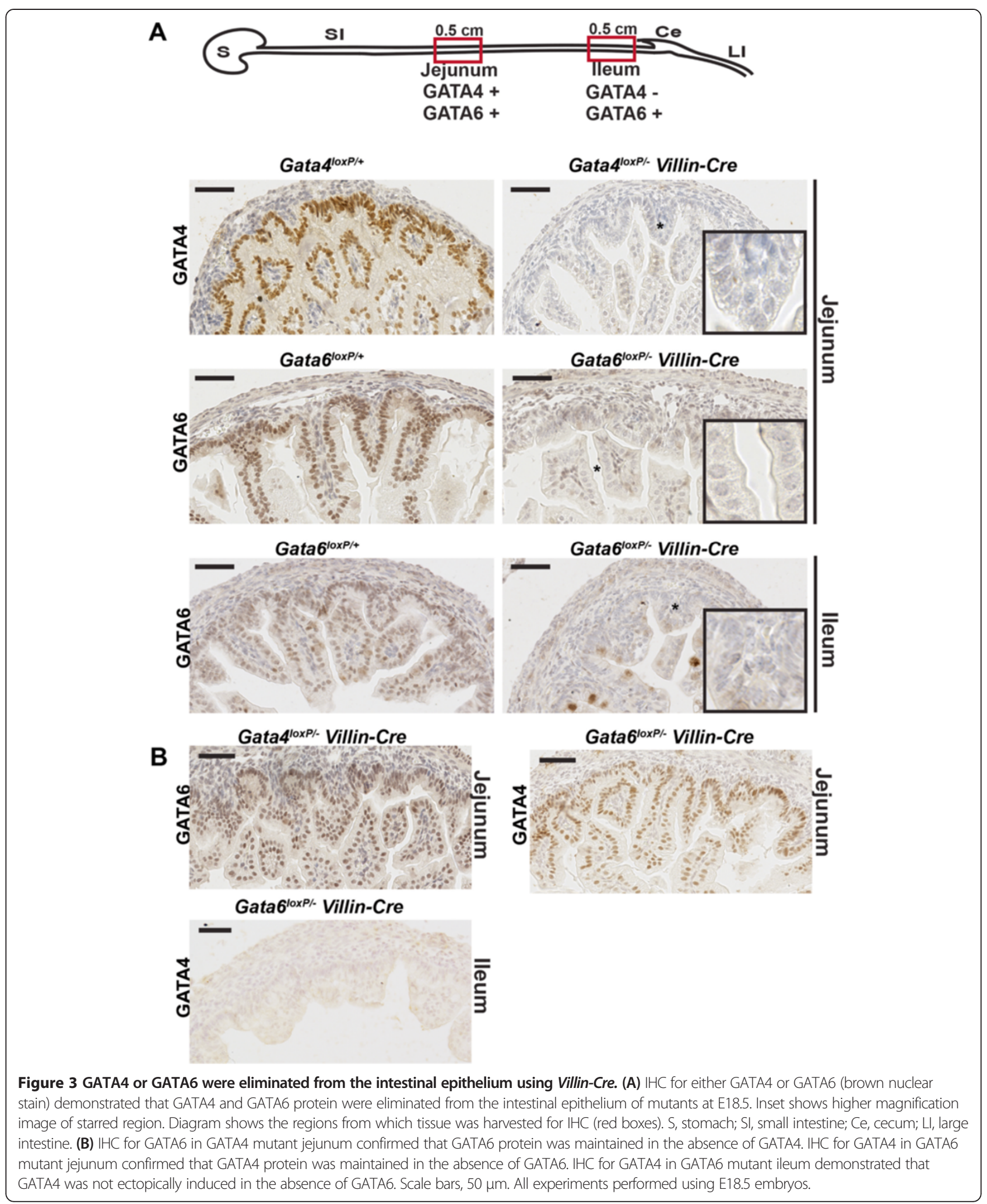

We further characterized specific intestinal epithelial cell types in GATA4 and GATA6 mutant jejunum using histochemistry and immunohistochemistry. To assess the enterocyte population, we stained for brush border alkaline phosphatase (AP) activity and found no difference between controls and GATA4 or GATA6 mutants 


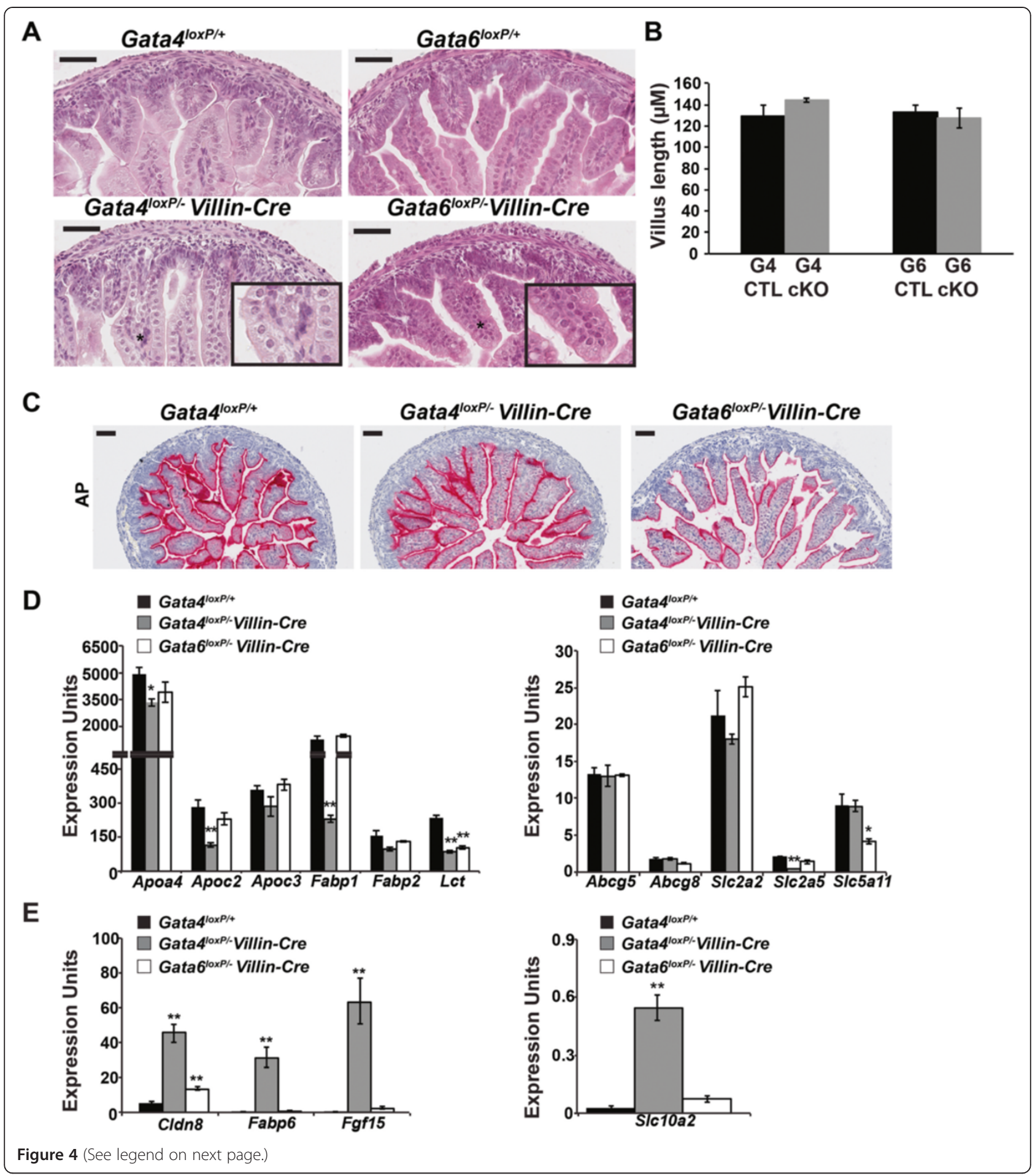


(See figure on previous page.)

Figure 4 GATA4 or GATA6 deletion in the jejunal epithelium perturbs enterocyte gene expression. (A) Hematoxylin and eosin (H\&E) staining of jejunal tissue from controls and GATA4 or GATA6 mutants showed no difference in intestinal architecture. (B) Average jejunal villus length was determined using NDP Scan software (Hamamatsu) to measure the length of all villi per section. No difference was observed between

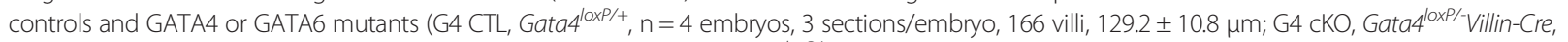
$\mathrm{n}=3$ embryos, 3 sections/ embryo, 132 villi, $144.1 \pm 1.8 \mu \mathrm{m} ; \mathrm{G} 6 \mathrm{CTL}$, Gata6 ${ }^{\text {loxP/+ }}$, $\mathrm{n}=4$ embryos, 3 sections/ embryo, 189 villi, $132.7 \pm 6.6 \mu \mathrm{m} ; \mathrm{G} 6 \mathrm{cKO}$,

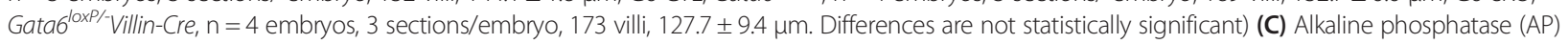
staining was equivalent between controls and mutants. (D) qRT-PCR for jejunal-enriched enterocyte transcripts (Apoa4, Apoc2, Apoc3, Fabp1, Lct, S/c2a2, S/c2a5, S/c5a11) showed that 5/8 were decreased in GATA4 mutants and that 2/8 were decreased in GATA6 mutants. None of the transcripts expressed at similar levels in jejunum and ileum (Fabp2, Abcg5, Abcg8) were changed in either mutant compared with controls. (E) qRT-PCR for ileal-enriched enterocyte transcripts (S/C10a2, Fabp6, Fgf15, Cldn8) showed that expression of each was induced in jejunum in the absence of GATA4. Only Cldn8 was induced in jejunum in the absence of GATA6. For qRT-PCR in D and E, epithelial cells from three control (Gata4 ${ }^{\text {loxP/+ }}$ ) and three mutant intestines

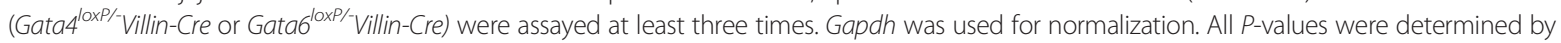
two-sample Student's $t$ test: ${ }^{*} P \leq 0.05,{ }^{* *} P \leq 0.01$. All error bars show SEM. Scale bars in all micrographs are $50 \mu \mathrm{m}$. All experiments performed using E18.5 embryos.

(Figure 4C) suggesting that loss of GATA4 or GATA6 does not alter jejunal enterocyte number.

Because the enterocyte gene expression profile of GATA4 mutant adult jejunum shifts from that of the jejunum toward that of the ileum [1], we performed qRT-PCR using epithelial cells from control and GATA4-deficient jejunum to compare expression of sets of jejunal-enriched transcripts (Apoa4, Apoc2, Apoc3, Fabp1, Lct, Slc2a2, Slc2a5, and Slc5a11), ileal-enriched transcripts (Slc10a2, Fabp6, Fgf15, and Cldn8), and transcripts expressed at similar levels in jejunum and ileum (Fabp2, Abcg5, and Abcg8). The majority of jejunal-enriched transcripts examined (5/8) were decreased in GATA4 mutant epithelium compared with control (Figure 4D). Moreover, expression of all ileal-enriched transcripts examined (4/4) was increased in GATA4 mutant epithelium compared with control (Figure 4E). Notably, ectopic expression of bile acid metabolism markers (Slc10a2, Fabp6, and Fgf15) was induced. None of the transcripts expressed ubiquitously in jejunum and ileum were changed in GATA4 mutant epithelium (Figure 4D). Taken together, these data suggest that gene expression changes in GATA4 mutant jejunal epithelium reflect a shift in jejunal enterocyte identity toward that of the ileum rather than a change in enterocyte cell number.

In contrast to GATA4-deficient jejunum, loss of GATA6 in adult mouse jejunum fails to alter enterocyte gene expression patterns [2]. We performed qRT-PCR for the same set of transcripts examined in GATA4 mutants using jejunal epithelial cells from control and GATA6-deficient jejunum. In general, gene expression profiles were conserved in jejunal epithelium lacking GATA6. However, we did find that expression of two jejunal-enriched transcripts, Lct and Slc5a11, and one ileal-enriched transcript, $C l d n 8$, was altered in GATA6 mutant epithelium (Figures 4D, E). These data suggest that loss of GATA6 during intestinal development subtly alters the jejunal enterocyte gene expression profile. Unlike GATA4-deficient jejunum, bile acid metabolism markers were not induced in jejunum lacking GATA6 suggesting that GATA4 uniquely regulates these genes in the developing jejunum.

To examine secretory cells in mutant jejunum, we stained for the goblet cell marker Mucin 2 (MUC2) and the pan-enteroendocrine cell marker Chromogranin A (CHGA). MUC2 staining revealed a 1.5-fold increase in goblet cells in GATA4-deficient jejunum and a 1.3-fold increase in goblet cells in GATA6-deficient jejunum compared with controls (Figure 5A,B). This agrees with our previously published observation that alcian blue positive goblet cells increase in the jejunum of Gata4 and Gata6 cKO embryos [12]. CHGA staining demonstrated a similar distribution of enteroendocrine cells within control and GATA4 or GATA6 mutant jejunum (Figure 5C). Because enteroendocrine cells constitute approximately $1 \%$ of the intestinal epithelium, we estimated enteroendocrine cell number using qRT-PCR to determine abundance of $C h g A$ and Ngn3. We found no change in the level of ChgA or Ngn3 transcripts (Figure 5D) suggesting that there is no alteration in enteroendocrine cell number GATA4 or GATA6 mutant jejunum. Examination of markers for specific enteroendocrine cell subpopulations, however, showed changes in enteroendocrine cell types present in GATA4 and GATA6 mutant jejunum (Figure 5D). GATA4deficient jejunal epithelium contained lower levels of Cck and Gip transcripts, which are markers of proximal intestine enteroendocrine cells. Abundance of Pyy transcript, which is a marker of distal intestine enteroendocrine cells, increased although this change was just outside of statistical significance $(\mathrm{P}=0.0744)$. Expression of these transcripts is similarly altered in the jejunum of adult Gata4 cKO mice [1]. Because GATA4 is not expressed in enteroendocrine cells [4], changes in enteroendocrine cell subtypes in GATA4-deficient jejunum likely reflect an indirect effect of GATA4. In contrast to adult GATA6-deficient jejunum, in which no change in enteroendocrine cell marker expression occurs [2], Pyy transcript abundance was decreased in jejunum of E18.5 Gata6 cKOs. Co-staining of tissue with 


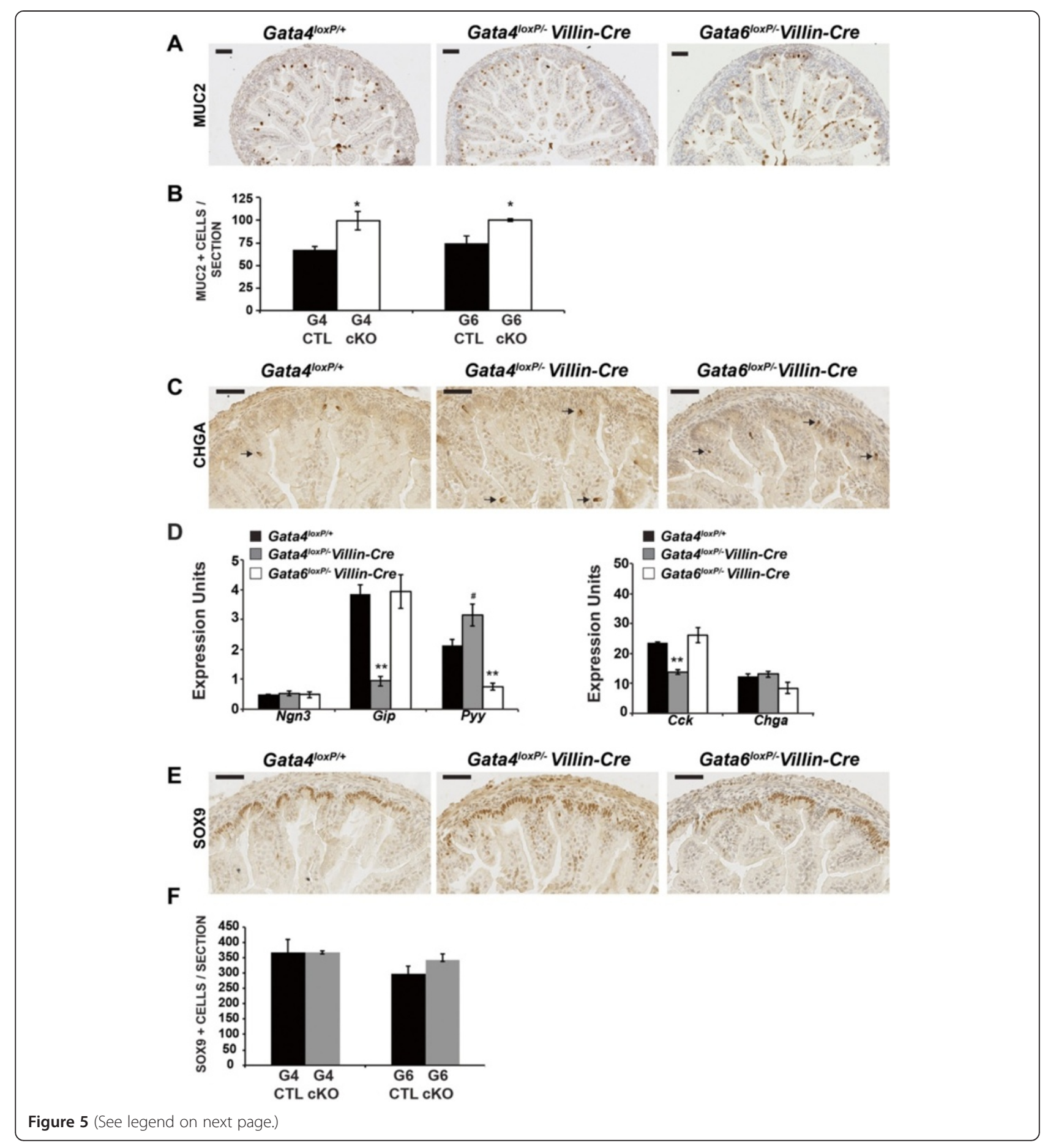


(See figure on previous page.)

Figure 5 GATA4 or GATA6 deletion in the jejunal epithelium alters goblet cell number and enteroendocrine cell gene expression. (A) MUCIN 2 (MUC2) IHC indicated a subtle increase in goblet cells in Gata4 and Gata6 cKO jejunum compared with controls. (B) Jejunum from Gata4 cKO and Gata6 cKO embryos contained more goblet cells than control jejunum (G4 CTL, Gata4 $4^{\text {loxP/4 }}, 68 \pm 4$ MUC2+ cells/section; G4 cKO,

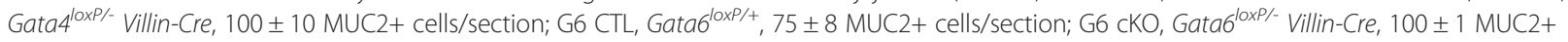
cells/section; $n=3$ embryos/genotype, 3 sections/embryo) (C) Chromogranin A (CHGA) IHC appeared comparable among jejunum from Gata4 CKO, Gata6 CKO, and control embryos. (D) qRT-PCR for enteroendocrine cell markers showed that pan-enteroendocrine cell marker expression (Ngn3, ChgA) was unchanged in the absence of GATA4 or GATA6. Expression of proximal enteroendocrine cell markers was decreased in GATA4 mutant jejunal epithelium (Gip, Cck). Expression of Pyy, a distally-enriched enteroendocrine cell marker, was increased in GATA4 mutants and

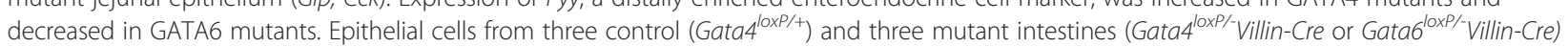
were assayed at least three times. Gapdh was used for normalization. (E) SOX9 IHC was unchanged between knockouts and controls. (F) Loss of

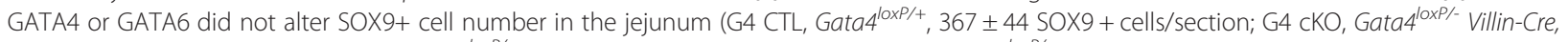

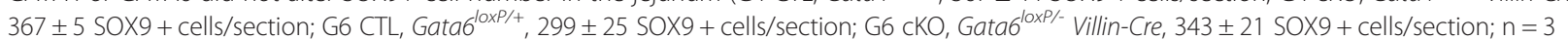
embryos/genotype, 3 sections/embryo; Differences were not statistically significant). P-values were determined by two-sample Student's $t$ test: ${ }^{*} P \leq 0.05,{ }^{* *} P \leq 0.01,{ }^{\#} P=0.0744$. Error bars show SEM. Scale bars are $50 \mu \mathrm{m}$. All experiments performed using E18.5 embryos.

antibodies to GATA6 and CHGA suggests that enteroendocrine cells express GATA6 (Additional file 2). Therefore, unlike GATA4 mutants, changes in abundance of enteroendocrine cell markers in GATA6 mutants may represent a cell autonomous phenotype. Moreover, it is interesting that loss of GATA4 increased Pyy expression whereas loss of GATA6 decreased Pyy expression. This difference may reflect direct regulation of Pyy transcription by GATA6 and indirect, non-cell autonomous regulation of Pyy by GATA4. Finally, we examined the intervillus region in jejunum of controls and GATA4 or GATA6 mutants by staining for the intervillus marker SOX9 and by counting SOX9+ cells. We found no difference in the localization or number of SOX9+ cells between groups suggesting that the intervillus region is normal in the absence of either GATA4 or GATA6 (Figure 5E,F).

To determine the effect of GATA6 loss on ileal epithelial development, we examined epithelial architecture in ileum of control and Gata6 cKO embryos at E18.5 using $\mathrm{H} \& \mathrm{E}$ staining (Figure 6A). We observed that villi in GATA6-deficient ileum were 26\% shorter compared with controls (Figure 6A). Villus length is also decreased when GATA6 is deleted within the ileum of adult mice using a tamoxifen-inducible Villin-Cre driver [2]. Because villus length was changed, we compared epithelial cell number in control and mutant tissue by staining with the pan-epithelial cell marker HNF4A and counting HNF4A+ cells per section. As expected and agreeing with Beuling et al. [2], we found a 1.7-fold decrease in epithelial cells in GATA6-deficient ileum compared with control ileum (Figure 6B). In contrast to the jejunum, in which loss of GATA4 affects villus length after birth [1] and loss of GATA6 fails to affect villus length [2], loss of GATA6 in the ileum affects villus length during development.

To assess the enterocyte population in GATA6 mutant ileum, we stained for brush border AP activity. We detected robust AP activity in control ileum whereas AP activity was very low or undetectable in GATA6deficient ileum (Figure 6C). This difference may reflect a decrease in enterocytes in mutant ileum or a shift in the identity of ileal enterocytes toward that of colonocytes as $\mathrm{AP}$ activity is normally lower in the colon compared with the small intestine [18]. A similar shift toward colon gene expression was observed when GATA6 is eliminated from the adult ileum [2]. If AP activity changes reflect decreased enterocyte number, we expected to see decreased enterocyte marker expression and no induction of colon markers. If AP activity reflects an identity change, we expected to see reduced enterocyte marker expression and induced colon marker expression. We observed the latter. Expression of the small intestine markers Fabp2 and Apoa4 was lost and expression of the colonic marker Car1 was induced in GATA6 mutants (Figure 6D). Moreover, expression of the intestinal alkaline phosphatase gene (Alpi) was lower in mutants compared with controls (Additional file 3). Taken together, these data suggest that enterocyte cell identity is shifted toward the colon in GATA6-deficient ileum.

To examine goblet cells in mutants, we stained for MUC2 and observed a 1.5-fold increase in goblet cells in GATA6 mutants compared with controls (Figure 7A). Goblet cells are also increased in the ileum of adult Gata6 cKOs although the increase is limited to the crypt, which does not form until after birth [2]. CHGA IHC identified enteroendocrine cells both in control and GATA6 mutant ileum (Figure 7B). We used ChgA qRTPCR as a measure of enteroendocrine cell number and found decreased ChgA expression in GATA6-deficient ileal epithelium compared with control epithelium (Figure 7B). The distal enteroendocrine cell marker Pyy was also decreased in GATA6-deficient ileum (Figure 7B). Together, these data suggest that loss of GATA6 in the ileum during development results in fewer enteroendocrine cells. This agrees with the findings of Beuling et al. [2] who showed decreased enteroendocrine cells in GATA6-deficient adult ileum. As GATA6 is expressed in enteroendocrine cells (Additional file 2), this may reflect a cell autonomous GATA6 phenotype. 


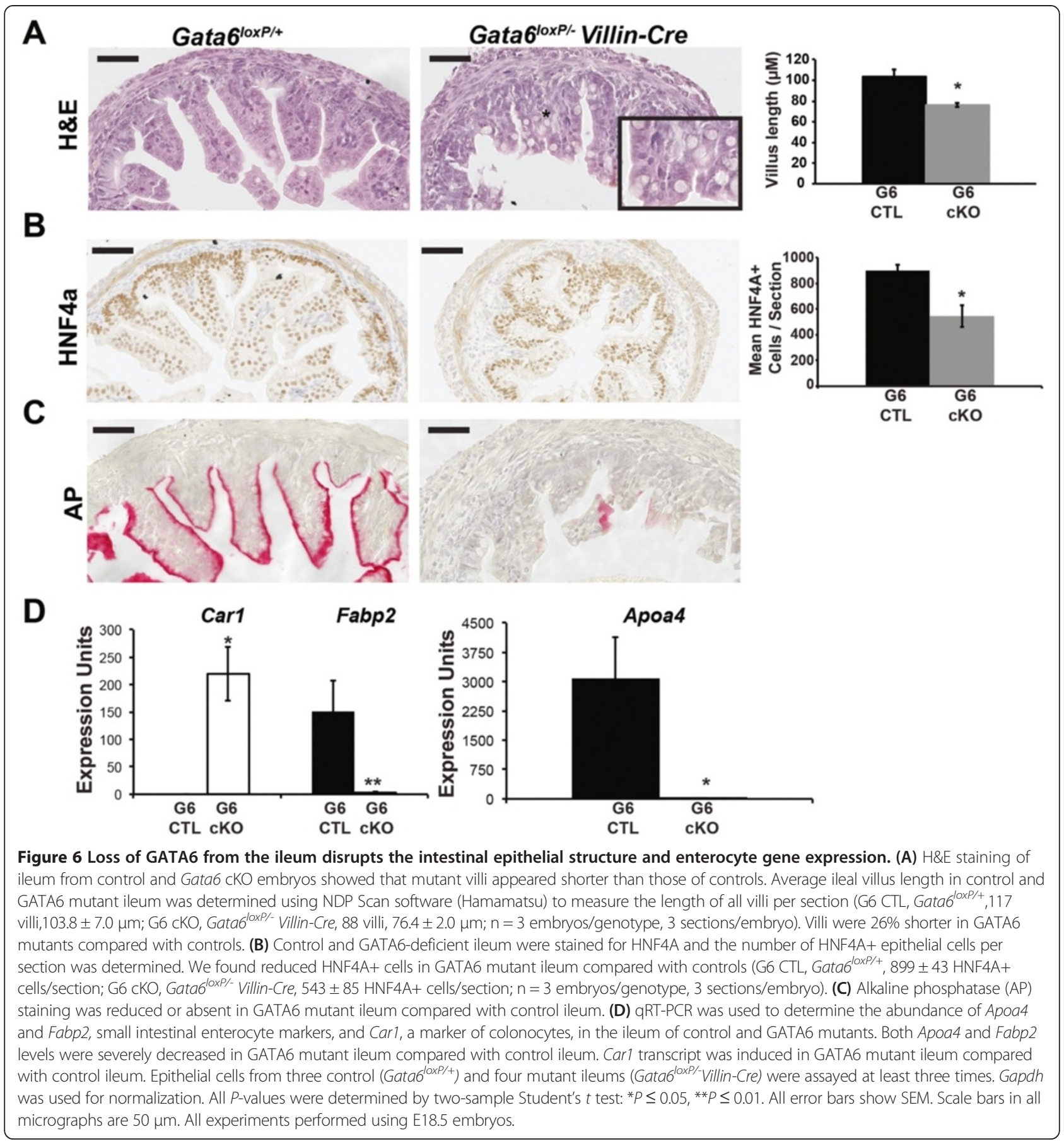

Finally, we examined the intervillus region in control and GATA6 mutant ileum by quantifying SOX9+ cell number (Figure 7C). We found a 1.3-fold increase in SOX9+ cells in GATA6-deficient ileum compared with control ileum suggesting that loss of GATA6 during ileal development causes an expansion of the intervillus region. As the intervillus region contains the proliferative progenitor population, we determined the number of proliferative cells in control and GATA6 mutant ileum by quantifying
KI67+ cells (Figure 7D). As predicted by SOX9 staining, we found a slight increase (1.2-fold) in KI67+ cells in GATA6-deficient ileum compared with control ileum. It is somewhat unexpected to find an increase in proliferative cells, yet fewer epithelial cells, in GATA6 mutant ileum. We examined cell death with cleaved caspase 3 IHC and observed only a few positive cells per section in both control and mutant ileum suggesting that increased cell death is not the reason for this disparity (Additional 


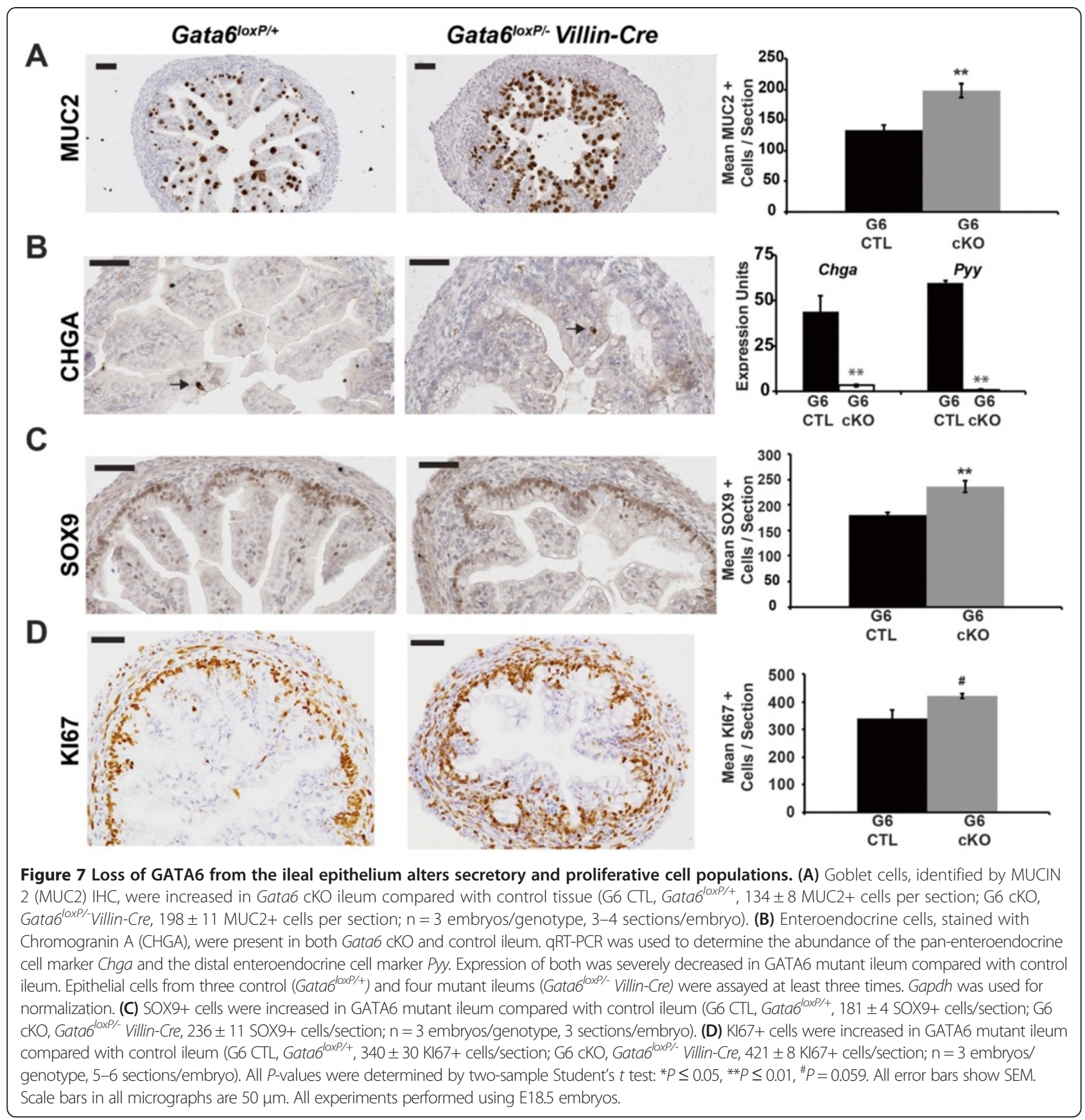

file 4). One alternative explanation is that increased proliferation occurs as a secondary consequence to compensate for epithelial cell loss and decreased villus length. We observed a similar effect when E-cadherin is deleted from the developing small intestine [19]. On the other hand, if this result reflects a direct effect of GATA6 loss, it would suggest that proliferation in the developing intestine is sensitive to total GATA level as we found no change in proliferation in either GATA4 or GATA6 mutant jejunum. When both GATA4 and GATA6 are deleted in the developing jejunum, however, proliferation is increased [12].
Finally, elimination of GATA6 from the adult ileum results in fewer proliferative cells in mature crypts [2]. This suggests that the requirement for GATA6 with respect to proliferation varies over time in the ileum.

\section{Conclusions}

This study investigated the impact of loss of either GATA4 or GATA6 within the intestinal epithelium during development. We found that intestinal development was generally normal in GATA4 and GATA6 mutants. Phenotypes observed in embryonic GATA4 or GATA6 mutant small 
intestine paralleled those identified in adult small intestine lacking either GATA4 or GATA6. The effect of loss of GATA6 in the developing ileum was greater than the effect of loss of either GATA4 or GATA6 in the developing jejunum. As the jejunum co-expresses GATA4 and GATA6 whereas the ileum expresses only GATA6, we conclude that overlapping GATA4 and GATA6 function in the jejunum lessens the effect of loss of either factor alone in this tissue. Although our data demonstrate redundancy between GATA4 and GATA6 function within the developing jejunum, examination of single knockouts suggests that GATA4 likely functions independently of GATA6 in the jejunum to regulate jejunal versus ileal enterocyte identity. Moreover, GATA6 likely plays a direct role in regulating enteroendocrine cell differentiation whereas GATA4 affects enteroendocrine cell development indirectly.

\section{Methods}

\section{Animals}

CD-1 mice (Charles River Laboratories, Wilmington, MA) were used to determine expression of GATA4 in embryonic small intestine. The Rosa26 conditional reporter strain Gt (ROSA)26Sor ${ }^{\text {tm1Sor }}$ (Rosa26R) was used to determine Cre activity [16]. Gata4 ${ }^{\text {tm1.1Sad }}$ (Gata4 ${ }^{\text {loxP }}$ ), Gata4 $4^{\text {tmolEno }}$ (Gata4 $4^{-}$), Gata6 $^{\text {tm2.1Sad }}$ (Gata6 ${ }^{\text {loxP }}$ ), Gata6 ${ }^{\text {tm2.2Sad }}\left(\right.$ Gata6 $^{-}$), and Tg (Vil-cre)997Gum (Villin-Cre) mice were used to generate

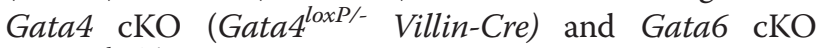

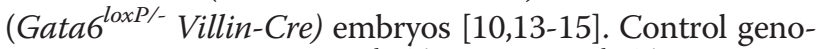
types were either Gata4 $4^{\text {loxP/+ }}$ or Gata6 ${ }^{\text {loxP/+ }}$. Embryos were obtained by timed mating with noon of the day of a vaginal plug considered as embryonic day (E) 0.5. Genotypes were determined by PCR using primers previously described [1,12]. The Medical College of Wisconsin's Animal Care Committee approved all animal procedures.

\section{Intestinal epithelial cell isolation}

Small intestine (jejunum or ileum) was isolated from E18.5 embryos, cut longitudinally, and incubated in cell dissociation buffer (BD Biosciences, San Jose, CA) for at least 6 hours at $4^{\circ} \mathrm{C}$ with shaking [20,21]. Mesenchymal tissue was removed, and epithelial cells were used to obtain total RNA for qRT-PCR experiments. Jejunal samples excluded epithelial cells from the distal 1-2 cm of the embryonic small intestine. Ileal samples contained epithelial cells from the distal $0.5-1.0 \mathrm{~cm}$ of the embryonic small intestine.

\section{Reverse transcription polymerase chain reaction}

DNase treated RNA was used to generate cDNA from isolated epithelial cells as previously described [19,22]. Additional file 5 contains a table listing the TaqMan assays identifiers used for qRT-PCR (Life Technologies, Carlsbad, CA). Gapdh was used as a normalization control.
Expression units for each target represent $\left(2^{-\Delta C q}\right) * 1000$. Multiplying by a factor of 1000 adjusted most expression unit values to $>1$. For all assays, gene expression was measured at least three times using cDNA generated from 3 control (Gata4 ${ }^{\text {loxP/+ }}$, jejunal assays; Gata6 $6^{\text {loxP/+ }}$, ileal assays) and 3-4 experimental (Gata4 ${ }^{\text {loxP/- } V i l l i n-C r e, ~ j e j u n a l ~ a s s a y s ; ~}$

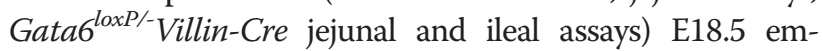
bryos. $P$-values were determined by a two-sample Student's $t$ test. Error bars represent standard error of the mean (SEM).

\section{Histochemistry and immunohistochemistry}

Histochemistry and immunohistochemistry were performed using previously described methods [19,22]. Jejunal tissue was harvested from the midpoint of the small intestine. The distal $0.5-1.0 \mathrm{~cm}$ of the embryonic small intestine was considered ileum for these assays. To assay $\beta$-galactosidase activity in tissue, embryonic intestine was dissected and fixed in $4 \%$ paraformaldehyde for 15 minutes at room temperature. Intestines were washed and incubated overnight in X-gal (5-bromo-4-chloro-3-indolyl- $\beta$-D-galactoside) stain as previously described [23]. Antibodies used for IHC are listed in Additional file 6.

\section{Additional files}

\begin{tabular}{|c|}
\hline $\begin{array}{l}\text { Additional file 1: qRT-PCR for Gata4 and Gata6 in conditional } \\
\text { knockout intestinal tissue. qRT-PCR was used to determine the } \\
\text { abundance of Gata4 and Gata6 mRNA in control and mutant jejunum and } \\
\text { ileum. For jejunal assays, epithelial cells from three control (Gata } 4^{\text {loxP/+ }} \text { ) and } \\
\text { three mutant jejunums (Gata } 4^{\text {loxP/- }} \text { Villin-Cre and Gata } 6^{\text {loxP/- }} \text { Villin-Cre) were } \\
\text { assayed at least three times. For ileal assays, epithelial cells from three } \\
\text { control (Gata } 6^{\text {loxP/+ }} \text { ) and four mutant ileums (Gata } 6^{\text {loxP/ }} \text { Villin-Cre) were } \\
\text { assayed at least three times. Gapdh was used for normalization. All P-values } \\
\text { were determined by two-sample Student's } t \text { test: }{ }^{*} P \leq 0.05 \text {. Error bars show } \\
\text { SEM. All tissue harvested was from E18.5 embryos. }\end{array}$ \\
\hline $\begin{array}{l}\text { Additional file 2: Immunohistochemical staining for GATA6 and } \\
\text { Chromogranin A in the mouse small intestine. E18.5 wild-type tissue } \\
\text { was co-stained for GATA6 (brown nuclear stain) and Chromogranin A } \\
\text { (CHGA; brown cytoplasmic stain) and counterstained with hematoxylin. } \\
\text { Higher magnification images of the boxed regions are shown at right. } \\
\text { Stars indicate GATA6+, CHGA+ enteroendocrine cells. Scale bar, } 50 \mu \mathrm{m} \text {. }\end{array}$ \\
\hline $\begin{array}{l}\text { Additional file 3: Semi-quantitative RT-PCR to determine the } \\
\text { expression level of the intestinal alkaline phosphatase gene (Alpi) in } \\
\text { control (Gata6 }{ }^{\text {IoxP/+ }} \text { ) and Gata6 cKO (Gata6 }{ }^{\text {IoxP/- Villin-Cre) ileal }} \\
\text { epithelium. Epithelial cells from three controls and four mutants were } \\
\text { assayed. PCR was performed including a32P-dATP. To quantify fold change, } \\
\text { a Phosphorimager (Molecular Dynamics) was used. Samples were } \\
\text { normalized to the level of Polr2a expression. Data shown are representative } \\
\text { of two experiments. Primer sequences are included in Additional file } 5 .\end{array}$ \\
\hline $\begin{array}{l}\text { Additional file 4: Cleaved caspase } 3 \text { immunohistochemistry in } \\
\text { control and GATA6 mutant ileum. E18.5 control (Gata6 }{ }^{l o x /+} \text { ) and GATA6 } \\
\text { mutant (Gata6 }{ }^{\text {loxP/- }} \text { Villin-Cre) ileum were stained for cleaved caspase } 3 \\
\text { (brown nuclear stain). Very few cleaved caspase } 3 \text { positive epithelial cells } \\
\text { were observed in ileum of either genotype ( } n=3 \text { embryos/genotype; } \\
3-4 \text { sections/embryo examined). Higher magnification images of the } \\
\text { boxed regions are shown as insets. Arrows indicate cleaved caspase } 3 \\
\text { positive cells. Staining from adult control tissue is shown as a positive } \\
\text { control for antibody staining. Several cleaved caspase positive cells are } \\
\text { present at the villus tip. }\end{array}$ \\
\hline
\end{tabular}


Additional file 5: TaqMan assays used for qRT-PCR. List of the identifiers for TaqMan primer/probe sets used in QRT-PCR analyses and primers used in semi-quantitative RT-PCR analyses.

Additional file 6: Antibodies. List of the antibodies used in study. Dilutions used, manufacturer, and catalog numbers are provided.

\section{Competing interests}

The authors declare that they have no competing interests.

\section{Authors' contributions}

EMW: Contributed to experimental design, performed the majority of the experiments, analyzed data, prepared manuscript draft. CAT: Analyzed expression of GATA4 in the embryonic small intestine, contributed to qRT-PCR experiments, prepared figures. BMK: Contributed to analysis of survival of Gata6 CKOs. MLF: Generated Gata6 cKO animals, performed genotyping of animals. MAB: Conceived and coordinated the project, contributed to experimental design and data analysis, prepared figures, and prepared final version of manuscript. All authors read and approved the final manuscript.

\section{Acknowledgments}

We thank Benjamin Bondow for technical assistance, Dr. Vivian Lee (Medical College of Wisconsin, Milwaukee, WI) for providing SOX9 antibody, Dr. Xiang-Xi (Mike) Xu (Miller School of Medicine, University of Miami, Miami, FL) for providing GATA6 antibody, and Dr. Jeffrey Molkentin (Cincinnati Children's Hospital Medical Center) for providing Gata4 null mice. We also thank Drs. Stephen Duncan and Allison Ebert (Medical College of Wisconsin, Milwaukee, WI) for helpful discussions and input into the manuscript.

Funding for this project was provided by grants from the US National Institutes of Health, National Institute of Diabetes and Digestive and Kidney Diseases (DK087873), the American Gastroenterological Association Foundation Research Scholar Award, and Advancing a Healthier Wisconsin to MAB.

Received: 18 August 2014 Accepted: 28 November 2014 Published: 11 December 2014

\section{References}

1. Battle MA, Bondow BJ, Iverson MA, Adams SJ, Jandacek RJ, Tso P, Duncan SA: GATA4 Is Essential for Jejunal Function in Mice. Gastroenterology 2008, 135:1676-1686.e1.

2. Beuling E, Baffour-Awuah NY, Stapleton KA, Aronson BE, Noah TK, Shroyer NF, Duncan SA, Fleet JC, Krasinski SD: GATA factors regulate proliferation, differentiation, and gene expression in small intestine of mature mice. Gastroenterology 2011, 140:1219-1229.e1.

3. Bosse T, Fialkovich JJ, Piaseckyj CM, Beuling E, Broekman H, Grand RJ, Montgomery RK, Krasinski SD: Gata4 and Hnf1alpha are partially required for the expression of specific intestinal genes during development. Am J Physiol Gastrointest Liver Physiol 2007, 292:G1302-G1314.

4. Bosse T, Piaseckyj CM, Burghard E, Fialkovich JJ, Rajagopal S, Pu WT, Krasinski SD: Gata4 is essential for the maintenance of jejunal-ileal identities in the adult mouse small intestine. Mol Cell Biol 2006, 26:9060-9070.

5. Koutsourakis M, Langeveld A, Patient R, Beddington R, Grosveld F: The transcription factor GATA6 is essential for early extraembryonic development. Development 1999, 126:723-732.

6. Molkentin JD: The zinc finger-containing transcription factors GATA-4, -5 , and -6 . Ubiquitously expressed regulators of tissue-specific gene expression. J Biol Chem 2000, 275:38949-38952.

7. Watt AJ, Zhao R, Li J, Duncan SA: Development of the mammalian liver and ventral pancreas is dependent on GATA4. BMC Dev Biol 2007, 7:37.

8. Fang R, Olds LC, Sibley E: Spatio-temporal patterns of intestine-specific transcription factor expression during postnatal mouse gut development. Gene Expr Patterns 2006, 6:426-432.

9. Kuo CT, Morrisey EE, Anandappa R, Sigrist K, Lu MM, Parmacek MS, Soudais C, Leiden JM: GATA4 transcription factor is required for ventral morphogenesis and heart tube formation. Genes Dev 1997, 11:1048-1060.

10. Molkentin JD, Lin Q, Duncan SA, Olson EN: Requirement of the transcription factor GATA4 for heart tube formation and ventral morphogenesis. Genes Dev 1997, 11:1061-1072.

11. Morrisey EE, Tang Z, Sigrist K, Lu MM, Jiang F, Ip HS, Parmacek MS: GATA6 regulates HNF4 and is required for differentiation of visceral endoderm in the mouse embryo. Genes Dev 1998, 12:3579-3590.
12. Walker EM, Thompson CA, Battle MA: GATA4 and GATA6 regulate intestinal epithelial cytodifferentiation during development. Dev Biol 2014, 392:283-294.

13. Madison BB, Dunbar L, Qiao XT, Braunstein K, Braunstein E, Gumucio DL: Cis elements of the villin gene control expression in restricted domains of the vertical (crypt) and horizontal (duodenum, cecum) axes of the intestine. J Biol Chem 2002, 277:33275-33283.

14. Sodhi CP, Li J, Duncan SA: Generation of mice harbouring a conditional loss-of-function allele of Gata6. BMC Dev Biol 2006, 6:19.

15. Watt AJ, Battle MA, Li J, Duncan SA: GATA4 is essential for formation of the proepicardium and regulates cardiogenesis. Proc Natl Acad Sci U S A 2004, 101:12573-12578.

16. Soriano P: Generalized lacZ expression with the ROSA26 Cre reporter strain. Nat Genet 1999, 21:70-71.

17. Thompson CA, Battle MA: Basic Science of Small Intestinal Development Translational Research and Discovery in Gastroenterology: Organogenesis to Disease. 2014:85.

18. Lev R, Griffiths WC: Colonic and small intestinal alkaline phosphatase. A histochemical and biochemical study. Gastroenterology 1982, 82:1427-1435.

19. Bondow BJ, Faber ML, Wojta KJ, Walker EM, Battle MA: E-cadherin is required for intestinal morphogenesis in the mouse. Dev Biol 2012, 371:1-12.

20. Li X, Madison BB, Zacharias W, Kolterud A, States D, Gumucio DL: Deconvoluting the intestine: molecular evidence for a major role of the mesenchyme in the modulation of signaling cross talk. Physiol Genomics 2007, 29:290-301.

21. Madison BB, Braunstein K, Kuizon E, Portman K, Qiao XT, Gumucio DL: Epithelial hedgehog signals pattern the intestinal crypt-villus axis. Development 2005, 132:279-289.

22. Duncan SA, Nagy A, Chan W: Murine gastrulation requires HNF-4 regulated gene expression in the visceral endoderm: tetraploid rescue of Hnf-4(-/-) embryos. Development 1997, 124:279-287.

23. Hogan B, Beddington R, Costantini F, Lacy E: Manipulating the Mouse Embryo: A Laboratory Manual, Hogan Manipulating the Mouse Embryo: A Laboratory Manual1994. Plainview, NY: ColdSpring Harbor Lab. Press; 1994.

doi:10.1186/1756-0500-7-902

Cite this article as: Walker et al:: Characterization of the developing small intestine in the absence of either GATA4 or GATA6. BMC Research Notes 2014 7:902.

\section{Submit your next manuscript to BioMed Central and take full advantage of:}

- Convenient online submission

- Thorough peer review

- No space constraints or color figure charges

- Immediate publication on acceptance

- Inclusion in PubMed, CAS, Scopus and Google Scholar

- Research which is freely available for redistribution 